\title{
Adsorbed and free lipid bilayers at the solid-liquid interface
}

\author{
T. Charitat ${ }^{1,2, a}$, E. Bellet-Amalric ${ }^{3, b}$, G. Fragneto ${ }^{3}$, and F. Graner ${ }^{1}$ \\ 1 Spectrométrie Physique ${ }^{c}$, Université Grenoble I, B.P. 87, 38402 St Martin d'Hères Cedex, France \\ 2 Université Grenoble I, LENSC, Maison des Magistères, 25 avenue des Martyrs, B.P. 166 X, 38042 Grenoble Cedex, France \\ 3 Institut Laue-Langevin, B.P. 156, 38042 Grenoble Cedex, France
}

Received 17 July 1998

\begin{abstract}
Single and double phosphocholine (DPPC and DSPC) bilayers adsorbed at the silicon-water interface have been prepared and characterised. The second bilayer, called "free bilayer", is a novel highly hydrated system floating at 20 to $30 \AA$ above the first one. Robust and reproducible preparation has been possible thanks to a combination of Langmuir-Blodgett and Langmuir-Schaeffer techniques. Carefully optimised neutron reflectivity measurements have allowed a precise non-destructive characterisation of the structure, hydration and roughness of the layers. This work opens new possibilities for the investigation of the interaction between membrane lipids and soluble proteins, in particular peptides too small to be visible with other techniques.
\end{abstract}

PACS. 68.55.Jk Structure and morphology; thickness - 81.15.Lm Liquid phase epitaxy; deposition from liquid phases (melts, solutions, and surface layers on liquids) - 61.12.Ha Neutron reflectometry

\section{Introduction}

Soft-matter physicists and biophysicists often look for ideal model lipid membranes [1] with apparently incompatible requirements such as known position and free fluctuations, high hydration and stability in time, large sample sizes at low costs, controlled structures and asymmetric lipid compositions, resistance to vibrations and no organic solvents. Most of those requirements are met by the new system we present in this paper.

We deposited a double phospholipid bilayer on a solid substrate in contact with water. The second bilayer, floating above the first one, was called "free bilayer at the solid-liquid interface". It was reproducibly prepared with a high coverage rate by mixing the standard LangmuirBlodgett [2] and Langmuir-Schaeffer [3] methods (Fig. 1). Single and double bilayers were deposited at surface pressures as low as 30 and $40 \mathrm{mN} / \mathrm{m}$ respectively.

A good local probe for in-plane imaging of phospholipid bilayers is atomic force microscopy in non-contact mode [4]. On the other hand, to obtain pertinent information at atomic scales on the structure of the layer normal to its plane, a technique routinely used consists in reflecting a radiation with a wavelength, $\lambda$, of a few angstroms. X-ray and/or neutron specular reflectivity have been extensively used in soft matter physics for stud-

\footnotetext{
a Present address: Institut Charles Sadron, 6 rue Boussingault, 67083 Strasbourg Cedex, France.

b e-mail: amalric@ill.fr

c CNRS-UMR 5588
}

ies of nanometer-thick monomolecular layers of phospholipid molecules at the air-water interface [5-8]. However, bilayers of phospholipids are barely stable at the air-water interface [9] and require hydrophilic media on both sides. A bilayer suspended in water is stable but position control is not sufficient for reflectivity measurements. The solution consists in grafting lipid layers on silicon [10], or physically adsorbing them on a hydrophilic oxide [11]. This allows an in-situ, non destructive investigation of thickness, roughness, hydration, fluctuations and composition of phospholipid bilayers in a liquid medium, as well as their interaction with proteins.

For these systems, neutrons [12] offer three main advantages over X-rays: they can cross condensed matter; the sample is not degraded; they are scattered by the atomic nucleus itself. Light chemical elements such as $\mathrm{C}, \mathrm{H}, \mathrm{O}$ or N, common in soft matter or biological systems, are easily detected by neutrons and not so well by $\mathrm{X}$-rays, since they have a small number of electrons. We show here that optimisation of the protocol and analysis reveals structural details with an angstrom resolution. As we demonstrate elsewhere [13], this is sufficient to detect modifications of the bilayer induced by short proteins, such as peptides of less than 20-30 amino-acids, too small to be directly detected by other techniques.

\section{Principles of specular reflectivity}

When two semi-infinite mediums are separated by a planar interface, a wave incident with an angle $\theta_{i n}$ is reflected along the specular direction $\theta_{\text {out }}=\theta_{\text {in }}$, as in optics. 

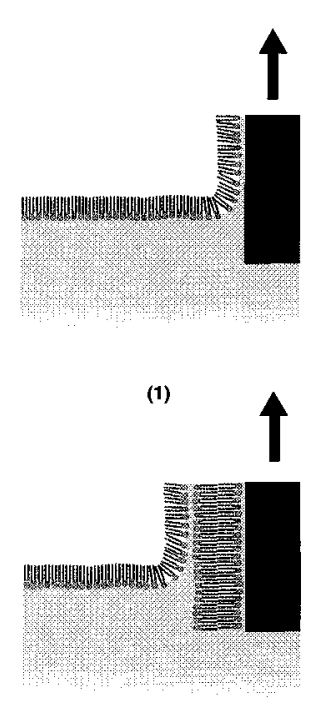

(3)

(a)

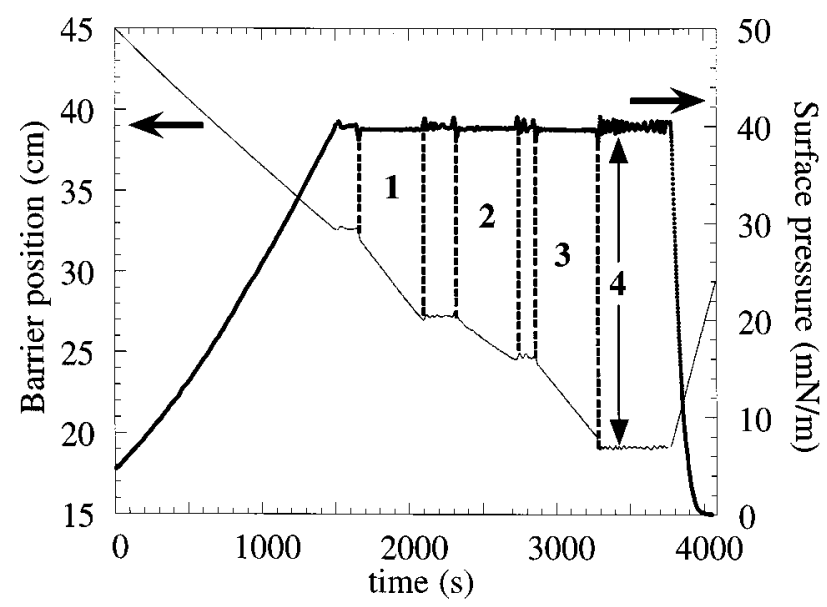

(b)

Fig. 1. (a) Schematic view of the successive deposition of a double bilayer. Monolayers 1, 2, 3 are deposited by the Langmuir-Blodgett method; the monolayer 4 is deposited by Langmuir-Schaeffer method. (b) Position of the barrier, $X$, and monolayer surface pressure, regulated at $40 \mathrm{mN} / \mathrm{m}$, versus time during the deposition of a DSPC sample. Same labels as in (a). Linear fits of $X(t)$ yield the slopes $d X / d t$, and thus the instantaneous transfer rates, here respectively $1.07 ; 0.6 ; 1.03$. The fourth layer is deposited at the time marked by arrows. An isotherm is recorded before and after the deposition to check the quality of the layer and the decrease in the number of molecules.

The component $q_{z}=2 \pi / \lambda\left(\sin \theta_{\text {in }}+\sin \theta_{\text {out }}\right)$ of the momentum transfer perpendicular to the interface is then equal to:

$$
q_{z}=(4 \pi / \lambda) \sin \theta_{i n}
$$

The amplitude of the reflected beam has the standard expression of Helmholtz equation, called the Fresnel reflectivity $R_{F}\left(q_{z}\right)$, which decays asymptotically as $q_{z}^{-4}$ when

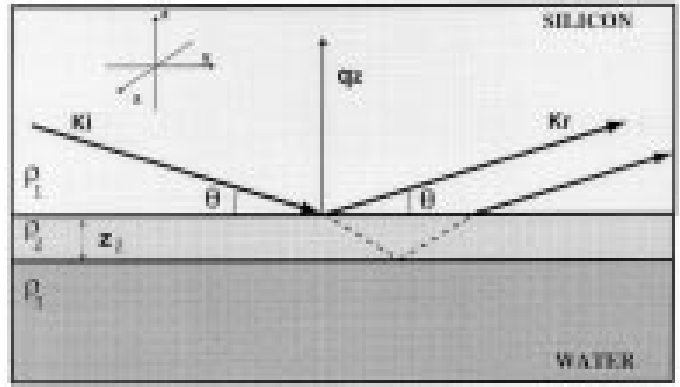

Fig. 2. The reflection of an incident beam from two ideally flat interfaces. $K_{i}$ and $K_{r}$ are the incident and scattered wave vectors, with angles $\theta_{\text {in }}=\theta_{\text {out }}=\theta$ in the incidence plane $y z$; $q_{z}$ is the component of the momentum transfer $q$ perpendicular to the interface; $\rho_{i}$ and $z_{i}$ are the mean scattering length density and the thickness of layer $i$. The neutrons hit the surface from the silicon side and are detected on a position-sensitive detector.

$q_{z}$ is larger than the critical momentum transfer $q_{c}$. Real interfaces are not flat at the nanometre scale, and part of the incident wave is in fact diffused in directions other than specular. The remaining specular reflectivity, dealt with in the present paper, falls off faster with a DebyeWaller like factor. All contributions to the roughness of the interface are lumped into a single number, the rms roughness $\sigma$ defined over the correlation area of the incident beam (here typically hundreds of microns). This approximation is discussed below, see [7] for a review.

If the system is composed of a stacking of several slabs of thickness $z_{i}$ separated by a planar interface, each one of constant refraction index (Fig. 2), the specular reflection includes a form factor due to the interference between the waves reflected at each interface. The calculation is standard, using as refraction index for neutrons $n_{i}^{2}=1-\left(\lambda^{2} / \pi\right) \rho_{i}$ where the neutron scattering length density $\rho_{i}$ is determined by the atomic nuclei in the slab $i$. In the limit of thin slabs the reflectivity function $R\left(q_{z}\right)$ can be expressed in terms of the Fourier transform of the scattering length density profile $\rho(z)$ along the normal $z$ to the interface:

$$
R\left(q_{z}\right)=R_{F}\left(q_{z}\right) e^{-\left(\sigma q_{z}\right)^{2}}\left|\frac{1}{\rho_{\infty}} \int_{-\infty}^{+\infty} d z \frac{\partial \rho}{\partial z} e^{i q_{z} z}\right|^{2} .
$$

Knowing only the modulus and not the phase of its Fourier components (Eq. (2)), the density profile $\rho(z)$ has to be guessed and fitted using either model-based approaches [12], or model-free approaches such as maximum entropy [14] and B-splines [15]. On phospholipid bilayers, Koening et al. [11] compared and mutually validated both approaches. Since isotopes have different scattering length densities, by repeating the same experiment on the same chemical system at different deuterium content, both of the material at the interface and of the bulk water, various samples become available which are physically almost identical, but have a very different refraction index for neutrons. This constrains the fits and allows to reliably extract the density profile. 
Table 1. Summary of the samples which reflectivity has been measured.

\begin{tabular}{ccccc}
\hline $\begin{array}{c}\text { lipid isotopic } \\
\text { composition }\end{array}$ & $\begin{array}{c}\text { deposition } \\
\text { pressure } \\
(\mathrm{mN} / \mathrm{m})\end{array}$ & $\begin{array}{c}\text { substrate } \\
\text { pre- } \\
\text { treatment }\end{array}$ & lid & water contrasts \\
\hline \multicolumn{5}{c}{ BILAYERS } \\
\hline h-DPPC & 30 & $\mathrm{UV} /$ ozone & teflon & $\mathrm{D}_{2} \mathrm{O} \mathrm{H}_{2} \mathrm{O} \mathrm{SMW}$ \\
& 30 & $\mathrm{UV} /$ ozone & teflon & $\mathrm{H}_{2} \mathrm{O} \mathrm{SMW}$ \\
d-DPPC & 30 & peroxide & glass & $\mathrm{D}_{2} \mathrm{O}$ \\
h-DSPC & 40 & peroxide & glass & $87 \% \mathrm{D}_{2} \mathrm{O}$ \\
\hline \multicolumn{5}{c}{ DOUBLE BILAYERS } \\
h-DPPC & 40 & peroxide & glass & $\mathrm{D}_{2} \mathrm{O}$ \\
\hline
\end{tabular}

\section{Experimental methods}

\subsection{Substrates}

The substrates were $5 \times 5 \times 1 \mathrm{~cm}^{3}$ single crystals of silicon. They were homogeneous and $n$-doped, poor in elements which scatter or absorb neutrons such as hydrogen or boron. The $5 \times 5 \mathrm{~cm}^{2}$ side, where the layer was deposited, was [111] oriented and had a radius of curvature $\geq 10^{3}$ m. It was "atomically smooth", with $\leq 5 \AA \mathrm{rms}$ roughness as determined by the polisher (Seso, Aix-en-Provence, France) by optical interferometry on a $300 \times 300 \mu \mathrm{m}^{2}$ portion of the surface: this is typically the scale of interest, since the correlation length of the neutron beam is of the order of hundreds of $\mu \mathrm{m}$. A high hydrophilicity was reached by using either of the two following oxidation techniques (see Tab. 1):

(i) a 10 minutes attack of native oxide [16] in $0.5 \%$ hydrofluoric acid, a test of the surface hydrophobicity, then a 15 minutes oxidation in $\mathrm{H}_{2} \mathrm{SO}_{4}: \mathrm{H}_{2} \mathrm{O}_{2}$ 12:1 vol:vol;

(ii) ozone: a 30 minutes exposure to a flow of oxygen under a UV lamp [17].

The final oxide had a constant thickness, as determined by ellipsometry and neutron reflectivity. The ozone method yielded a stable and reproducible oxide; immediately before each new deposition, substrates were cleaned in solvents (chloroform, acetone, alcohol, alcohol + water, and water) and again treated with ozone for $30 \mathrm{~min}$ in order to remove possible organic contaminants: the thickness of the oxide was not modified, indicating that passivation was good. Hydrophilicity was excellent and homogeneous, as determined in statics by size and wetting angle of pure water drops, and in dynamics by irisation (equal thickness) fringes during water drainage. This is probably due to the replacement of all $\mathrm{H}$ terminations by $\mathrm{OH}$ terminations [16]. We performed different controls of the roughness of parts of the substrates before or after oxidation at scales ranging from the millimetre to the centimetre, including profilometry (Talistep) before and after oxidation, atomic force microscopy after oxidation, neutron reflectivity after oxidation.

\subsection{Chemicals}

Phosphatidylcholine (hereafter PC) heads and saturated tails were chosen for their dominant presence amongst membrane lipids and their chemical stability, respectively. DPPC has 16 carbons per tail chain; DSPC has 18 carbons and forms thicker layers which are more visible in reflectivity. Protonated $L-\alpha$ Di-stearyl phosphatidyl-choline $(h$-DSPC) from Sigma, protonated and deuterated $(d-75)$ $L$ - $\alpha$ Di-palmitoyl phosphatidyl-choline $(h$-DPPC and $d-$ DPPC, respectively) from Avanti Polar Lipids were purchased as powder, dissolved in chloroform and stored without further purification. The four water subphases had scattering length densities in roughly arithmetical progression $0 / 2 / 4 / 6$ :

(i) $\mathrm{H}_{2} \mathrm{O}(18 \mathrm{M} \Omega . \mathrm{cm}$, Elga), neutron scattering length density of $-0.56 \times 10^{-6} \AA^{-2}$;

(ii) $\mathrm{SMW}=$ silicon-matched water, a mixture of $\mathrm{H}_{2} \mathrm{O}$ and $\mathrm{D}_{2} \mathrm{O}$ with the same refraction index for neutrons as bulk silicon, that is scattering length density of $2.07 \times 10^{-6} \AA^{-2}$;

(iii) $4 \mathrm{MW}=$ four-matched water, a mixture of $\mathrm{H}_{2} \mathrm{O}$ and $\mathrm{D}_{2} \mathrm{O}$ with neutron scattering length density of $4 \times 10^{-6} \AA^{-2}$;

(iv) $\mathrm{D}_{2} \mathrm{O}$, density $1.10445 \mathrm{~g} \mathrm{~cm}^{-3}$ at $25{ }^{\circ} \mathrm{C}$, purified at the ILL, neutron scattering length density of $6.35 \times 10^{-6} \AA^{-2}$.

\subsection{Single bilayer depositions}

The home-made teflon Langmuir trough had a volume $50 \times 10 \times 0.3 \mathrm{~cm}^{3}$ and a well to dip the substrate. The movement of a teflon barrier yielded a compression ratio around 5. A box ensured its thermalisation at $24{ }^{\circ} \mathrm{C}$ and protection against evaporation. Surface tension was measured with a Wilhelmy balance (Riegler \& Kirstein). The whole setup was kept clean in an over-pressurised horizontal flux hood. 
Lipid molecules were deposited on the subphase, until their surface pressure became measurable $\left(\geq 0.2 \mathrm{mN} / \mathrm{m}\right.$ i.e. about $10 \mathrm{nmol}$ over $\left.45 \times 10 \mathrm{~cm}^{2}\right)$, and equilibrated for half an hour to allow for solvent evaporation. A compression-decompression isotherm was recorded to check the quality of the monolayer. After a new compression, the surface pressure was regulated within $\pm 0.1 \mathrm{mN} / \mathrm{m}$ at the value chosen for deposition. The substrate was dipped into the water with the large face vertical (Fig. 1). As expected for a clean hydrophilic substrate, no hydrophobic tail adsorbed, so that the barrier moved to compensate exactly this suppression of surface.

The first monolayer was deposited according to the standard Langmuir-Blodgett method. The substrate was lifted upwards at a speed of $0.5 \mathrm{~cm} / \mathrm{min}$. This is an optimal deposition speed, according to the literature [18-20] and our own tests: slow enough to allow for water drainage, fast enough to prevent the formation of holes in the layers. Irisation fringes of equal thickness were clearly visible, parallel to the water surface, indicating that the substrate was defect-free; their spacing was stationary in time, showing that the draining meniscus was in a stationary regime. The surface pressure was kept constant and the barrier moved at constant velocity. The ratio of the barrier and substrate velocities measured the lipid molecules instantaneous transfer rate on the substrate. It was $1.09 \pm 0.01$ in average over the 24 samples analysed by neutron reflectivity (described here and in [13]) and did not correlate with the composition or the surface pressure of the lipid monolayer. While we expected a value of 1 for a perfect transfer, a value slightly higher than 1 might simply indicate that equilibrium packing of lipids is denser on silicon surface than on water. We measured also the global transfer rate from the total displacements of barrier and substrate between the equilibrium states at the beginning and the end of the deposition, after corrections due to geometry and kinetics. It was equal to $0.97 \pm 0.01$ and lower by 0.12 than, but correlated with, the instantaneous transfer rate.

The last monolayer was deposited by using the Langmuir-Schaeffer technique. The substrate was rotated so that the polished face was almost horizontal (Fig. 1): a small inclination $\left(<1^{\circ}\right)$ allowed for a good wetting free of air bubbles when the substrate was slowly brought into contact with the monolayer on the water surface. The barrier regulated the pressure back to its assigned value within ten seconds; its displacement indicated the global transfer rate. At surface pressure as low as $30 \mathrm{mN} / \mathrm{m}$, the transfer rate was $0.9 \pm 0.1$. At higher surface pressures, up to collapse, the transfer rate was the same [4]. At lower surface pressures, down to $15 \mathrm{mN} / \mathrm{m}$, the transfer rate significantly decreased. We thus decided to deposit both monolayers at the same pressure of $30 \mathrm{mN} / \mathrm{m}$. Data regarding samples with higher pressures, mixed lipids or buffered subphase are presented elsewhere [13].

After the deposition of the last monolayer, the substrate was pushed through the surface onto a clean glass lid, where a $0.3 \mathrm{~mm}$ deep trough had been machined. The small volume of enclosed water protected the lipid layers;

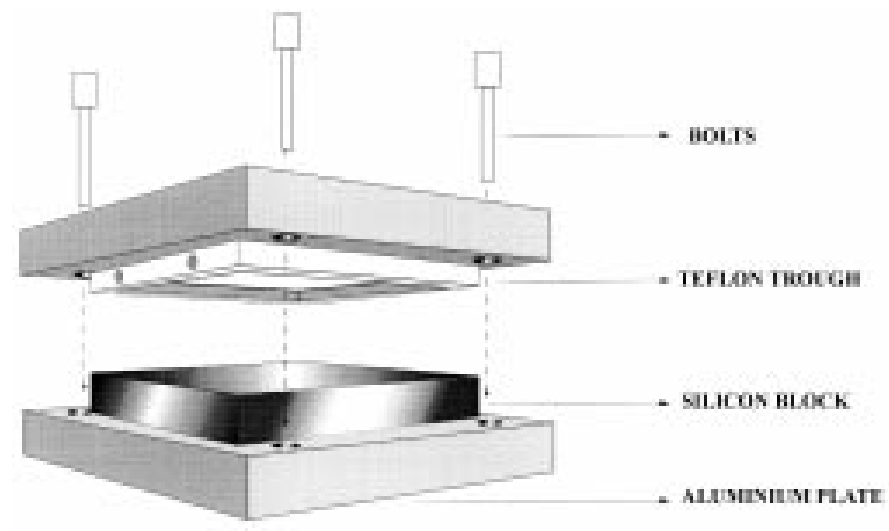

Fig. 3. Schematic representation of the cell. The single or double bilayer deposited on the surface of a silicon crystal is in contact with a $\mathrm{H}_{2} \mathrm{O} / \mathrm{D}_{2} \mathrm{O}$ mixture subphase enclosed by a PTFE or glass lid, respectively 1 and $0.3 \mathrm{~mm}$ deep.

the absence of air bubbles was checked visually through the glass. Later, in order to rinse the sample with different subphases without removing it from the neutron beam, the glass lid was later opened under water and replaced by a PTFE trough where two holes had been drilled (Fig. 3).

Various tests of the quality of the final samples were performed:

(i) Ellipsometry on one layer at the silicon-air interface, and on two layers at the silicon-water interface. The measured thickness was compatible with the number of expected layers. Homogeneity was checked on the scale of the light beam size, i.e. a millimeter;

(ii) Microscopy on partially fluorescent monolayers or trilayers of lipids, at the glass-air interface (and not silicon-air interface [21]). The coverage was good and almost homogeneous on the micrometre scale;

(iii) Atomic force microscopy on two layers at siliconwater interface. Bilayer-deep holes, sub-micrometric in diameter (similar to those observed on mica [19]), covered a fifth of the surface;

(iv) Neutron reflectivity. The observed structure was identical for different sample depositions in identical conditions, after manipulations and several rinsings.

\subsection{Double bilayer depositions}

For double bilayers, the second and third monolayer were prepared according to the classical Langmuir-Blodgett deposition on a vertical substrate (Fig. 1).

The second monolayer was thus formed at nearequilibrium, ensuring a stable deposition of the next monolayers; this would not be true with the LangmuirSchaeffer method. At $40 \mathrm{mN} / \mathrm{m}$ we obtained an instantaneous transfer rate of $0.7 \pm 0.1$, which is the optimum within our goals and is considered satisfactory for phosphocholines heads [20]. At higher surface pressures, up to collapse, the transfer rate was the same. At lower surface pressures deposition was not possible $[18,19]$. We thus 


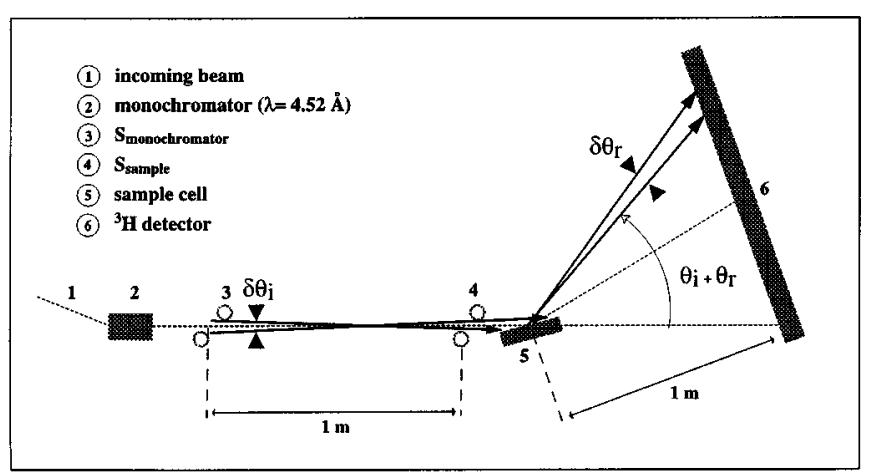

Fig. 4. Layout of the diffractometer D16 at the ILL, see text for details.

decided to deposit the four successive monolayers at the same pressure of $40 \mathrm{mN} / \mathrm{m}$.

The third monolayer yielded the same transfer rate as the first one. This indicated that, although the first bilayer was incomplete, the entire surface was hydrophilic, in agreement with the picture of patches of bare substrate within an almost complete surface of hydrophilic lipid heads [19].

To deposit the fourth monolayer, the block was rotated in the Langmuir-Schaeffer configuration with a global transfer rate of $0.9 \pm 0.1$, as for a single bilayer, and pushed through the water surface onto the same glass lid. Note that, as expected, we could not deposit the fourth layer in the vertical Langmuir-Blodgett configuration: the second bilayer, much less anchored than the first one, systematically floats off on water whatever the surface pressure [22].

\subsection{Reflectivity setup}

Measurements were performed at the Institut Laue Langevin (Grenoble, France) on a modified version of the D16 diffractometer (Fig. 4). Cold neutrons of wavelength $4.52 \AA$ were selected by a pyrolytic graphite monochromator $(\Delta \lambda / \lambda=1 \%)$. The flux at the sample position, $\sim 10^{6}$ neutron $\mathrm{cm}^{-2} \mathrm{~s}^{-1}$, was monitored by a low efficiency detector to normalise the reflected intensities. The neutron beam entered the silicon substrate through a $5 \times 1 \mathrm{~cm}^{2}$ side, hit at grazing incidence the polished $5 \times 5 \mathrm{~cm}^{2}$ face on which the layer under study had been deposited, and went out through the opposite $5 \times 1 \mathrm{~cm}^{2}$ side. The diminution in intensity due to scattering on $5 \mathrm{~cm}$ of silicon was $17 \%$.

For each experimental point the sample was positioned at an angle $\theta_{\text {in }}$ (precision 0.005 degrees) with respect to the direct beam. Neutrons were detected at $1 \mathrm{~m}$ from the sample by a planar bidimensional ${ }^{3} \mathrm{He}$ neutron counter of 16 horizontal wires (covering a $4.5^{\circ}$ angle in the vertical direction) and 64 vertical wires (covering a $9^{\circ}$ angle). The detector efficiency was calibrated wire by wire by using the scattering of $1 \mathrm{~mm}$ of $\mathrm{H}_{2} \mathrm{O}$ in a quartz cell, incoherent and constant in the angular range measured. At the

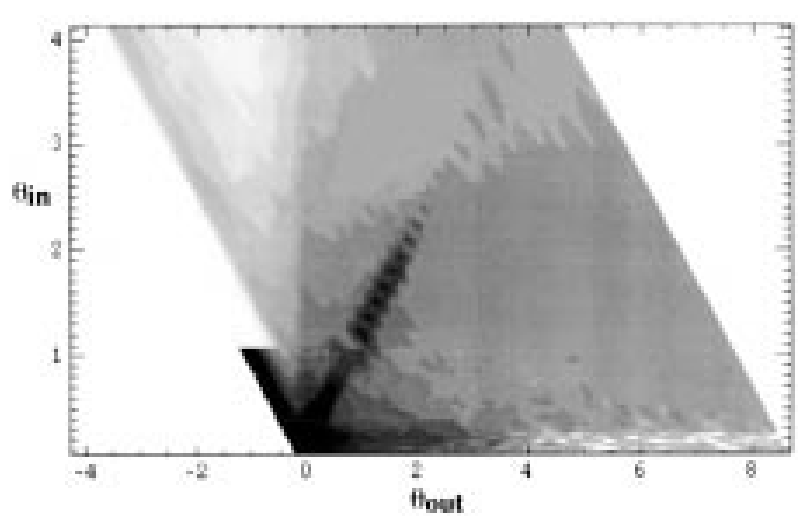

Fig. 5. Reflectivity $\mathrm{R}$ of a DSPC double bilayer in $\mathrm{D}_{2} \mathrm{O}$, here as a two-dimensional plot in the incidence plane. For each value of $\theta_{i n}$, raw data was collected by the detector for different $\theta_{\text {in }}+\theta_{\text {out }}$ values; $\theta_{\text {in }}=\theta_{\text {out }}$ corresponds to the specular direction. $\operatorname{Ln} R\left(\theta_{\text {in }}, \theta_{\text {out }}\right)$ is represented in grey levels without any background substraction. The neat separation for angles $\theta_{\text {in }}$ greater than 0.145 degrees, corresponding to the critical angle $\mathrm{Si} / \mathrm{D}_{2} \mathrm{O}$, shows the position of one of the Yoneda's peak. The neat separation for angles $\theta_{\text {out }}$ greater than 0.145 degrees reveals the position of the other Yoneda's peak. Near $\theta_{\text {in }}+\theta_{\text {out }}=0$, the foot of the direct beam is visible (in black) up to $\theta_{\text {in }}=1^{\circ}$. Above $\theta=1^{\circ}$ the collimating slit has been opened and a beam stop made of cadmium has been interposed to prevent detector saturation.

beginning of a set of experiments, the detector was centred around $\theta_{\text {out }}=0$ for the measurement of the direct beam. The detector was then placed and kept fixed in order to record simultaneously the intensity over the whole 0-4 degrees range of $\theta_{\text {out }}$, which corresponds to the measurable wave-vector domain, essentially limited by the signal over noise ratio. In the scattering plane the resolution on $\theta_{\text {out }}$, defined by the distance between detection wires, was 0.145 degrees. This resolution could not be improved by displacing the detector, due to the mechanical precision of its movement. For further details see Appendix.

Figure 5 presents an example of raw data $R\left(\theta_{\text {in }}, \theta_{\text {out }}\right)$, measured on a double bilayer of DSPC. This twodimensional plot displays all main features of reflectivity experiments:

(i) The first diagonal represents the specular reflection peaks $\theta_{\text {in }}=\theta_{\text {out }}$, here measured at discrete positions;

(ii) On each side of these peaks in the whole region $\theta_{\text {in }}>$ $\theta_{c}, \theta_{\text {out }}>\theta_{c}$ limited by the critical angles, is the offspecular diffuse reflection;

(iii) At the critical angles themselves, respectively $\theta_{i n}=$ $\theta_{c}$ and $\theta_{\text {out }}=\theta_{c}$, both Yoneda peaks [23], due to the roughness of the double bilayer, are (slightly) visible. On a single bilayer, the Yoneda peaks were not visible, unless it was deposited on a rough substrate [unpublished data];

(iv) Beside the critical angles, in the region $\theta_{i n}<\theta_{c}$, is the background noise due to incoherent neutron scattering. 
Table 2. Parameters derived from model fitting the curves measured from the bilayers. SLD stands for scattering length density. Errors indicate the incertitude on the fit. Note that the variations in water amount, and in SLD of heads and tails, remain within error bars.

\begin{tabular}{lcccccc}
\hline \multicolumn{1}{c}{ layer } & oxide & $\begin{array}{c}\text { water } \\
\text { layer 1 }\end{array}$ & heads & chains & heads & error \\
& \multicolumn{7}{c}{ DPPC BILAYER } \\
\hline thickness $(\AA)$ & 10 & 8 & 8 & 32 & 8 & \pm 1 \\
SLD $\left(10^{-6} \AA^{-2}\right)$ & 3.41 & & 1.5 & -0.4 & 1.5 & \pm 0.2 \\
\% water & 0 & 100 & & 20 & & \pm 3 \\
roughness $(\AA)$ & 4 & 4 & 4 & & \pm 1 \\
\hline \multicolumn{7}{c}{ DSPC BILAYER } \\
\hline thickness $(\AA)$ & 13 & 7 & 8 & 35 & 8 & \pm 1 \\
SLD $\left(10^{-6} \AA^{-2}\right)$ & 3.41 & & 1.5 & -0.9 & 1.5 & \pm 0.5 \\
\% water & 0 & 100 & & 15 & & \pm 5 \\
roughness $(\AA)$ & 6 & 6 & & 7 & & \pm 2 \\
\hline
\end{tabular}

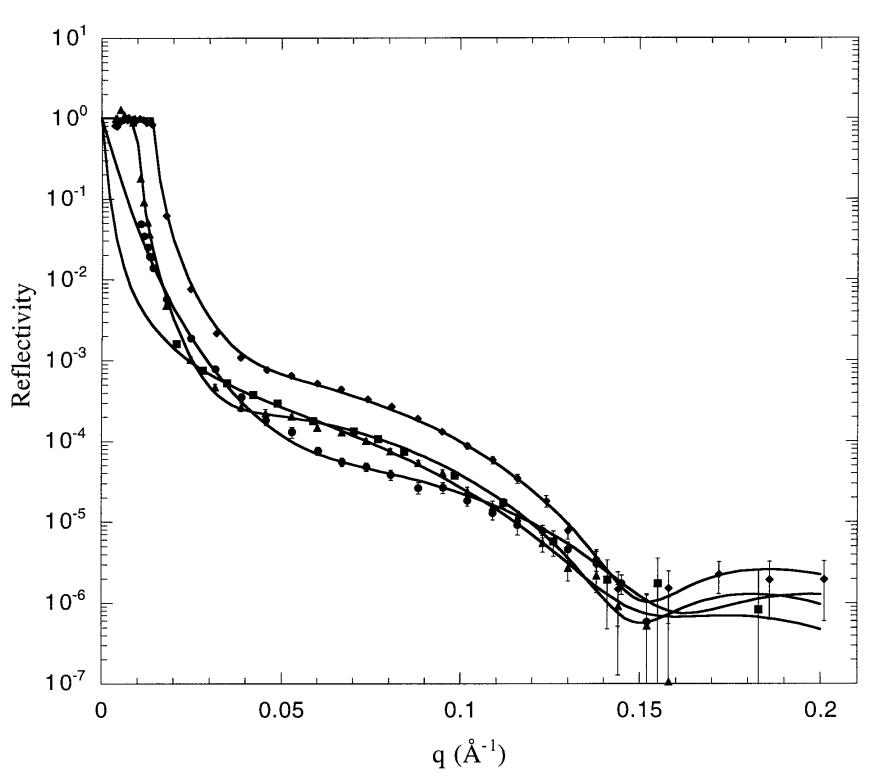

Fig. 6. Specular reflectivity profiles $R\left(q_{z}\right)$ of a bilayer of protonated DPPC in four water contrasts: $(\bullet) \mathrm{D}_{2} \mathrm{O},(\boldsymbol{\Delta}) 4 \mathrm{MW}$, (ם) SMW, and $(\bullet) \mathrm{H}_{2} \mathrm{O}$. Solid lines are best fits by parameters listed in Table 2. On this figure and on the next one, errors bars indicate a $90 \%$ confidence level.

\section{Results}

\subsection{Single bilayers}

The structure of DPPC bilayers was determined with high resolution by using four different deuterations of the water subphase, and hydrogenated or deuterated lipid tails (Tab. 1). Examples of specular reflectivity curves are presented in Figure 6. Their oscillations were clearly visible and reflectivity was measured down to $5 \times 10^{-7}$. The values of thicknesses, roughnesses, and scattering length densities found to best fit the curves are presented in Table 2 :

- The total thickness of the deposited bilayer was $49 \pm 1 \AA$.
- The bilayer was resolved into heads-tails-heads boxes, each within a $1 \AA$ resolution; these incertitudes were not independent since their sum is known.

- We found that those three boxes contained the same proportion of water, around $20 \%$ vol:vol.

- There was a $8 \AA$ thick film of $100 \%$ water between the substrate and the bilayer.

- Replacing DPPC by DSPC resulted in reflectivity measurements down to $6 \times 10^{-7}$. A marked oscillation at $0.143 \AA^{-1}$ constrained the fit although only one contrast $\left(\mathrm{D}_{2} \mathrm{O}\right)$ has been measured. As expected, all results were the same as with DPPC within error bars, except for

(i) the lipid tails box was $2 \pm 1 \AA$ larger, and

(ii) the oxide thickness and roughness of the substrate used were larger, which increases accordingly the roughness of the bilayer itself.

\subsection{Double bilayers}

Measurements were performed on a fresh DSPC sample, on a 24 hours old DPPC sample, and on the same DPPC sample after 24 additional hours. Results presented in Figure 7 and Table 3 show that the structure was that of a double bilayer, with the following characteristics:

- Each bilayer had a head-tails-head structure comparable to the single bilayer previously investigated.

- The first bilayer was firmly adsorbed on, and slightly rougher than, the substrate. The second bilayer, more free to fluctuate, was significantly $(5-6 \AA)$ rougher than the substrate.

- There was a clear hydration film between the lipid heads of the first and second bilayer. Its thickness was pretty well determined, since the oscillations of the reflectivity curve strongly constrained the total thickness of the whole system (bilayer-water-bilayer). Our assumptions on each bilayer structure thus yielded a precision of $1 \AA$ on the water film thickness: $20 \pm 1 \AA$ thick for the fresh DSPC sample, and $29 \pm 1 \AA$ for both aged DPPC samples. 
Table 3. Same as Table 2 for the double bilayers.

\begin{tabular}{|c|c|c|c|c|c|c|c|c|c|c|}
\hline layer & oxide & $\begin{array}{c}\text { water } \\
\text { layer } 1\end{array}$ & heads & chains & heads & $\begin{array}{c}\text { water } \\
\text { layer } 2\end{array}$ & heads & chains & heads & error \\
\hline \multicolumn{11}{|c|}{ DPPC DOUBLE BILAYER } \\
\hline thickness $(\AA)$ & 19 & 0 & 8 & 34 & 9 & 29 & 8 & 34 & 9 & \pm 1 \\
\hline $\operatorname{SLD}\left(10^{-6} \AA^{-2}\right)$ & 3.41 & & 1.5 & -0.4 & 1.5 & & 1.5 & -0.4 & 1.5 & \pm 0.2 \\
\hline$\%$ water & 0 & & & 15 & & 100 & & 18 & & \pm 3 \\
\hline roughness $(\AA)$ & 12 & & & 15 & & 18 & & 18 & & \pm 3 \\
\hline \multicolumn{11}{|c|}{ DSPC DOUBLE BILAYER } \\
\hline thickness $(\AA)$ & 14 & 0 & 8 & 36 & 9 & 20 & 8 & 36 & 9 & \pm 1 \\
\hline $\operatorname{SLD}\left(10^{-6} \AA^{-2}\right)$ & 3.41 & & 1.6 & -0.6 & 1.6 & & 1.6 & -0.6 & 1.6 & \pm 0.2 \\
\hline$\%$ water & 0 & & & 22 & & 100 & & 17 & & \pm 3 \\
\hline roughness $(\AA)$ & 8 & & & 8 & & 12 & & 13 & & \pm 3 \\
\hline
\end{tabular}
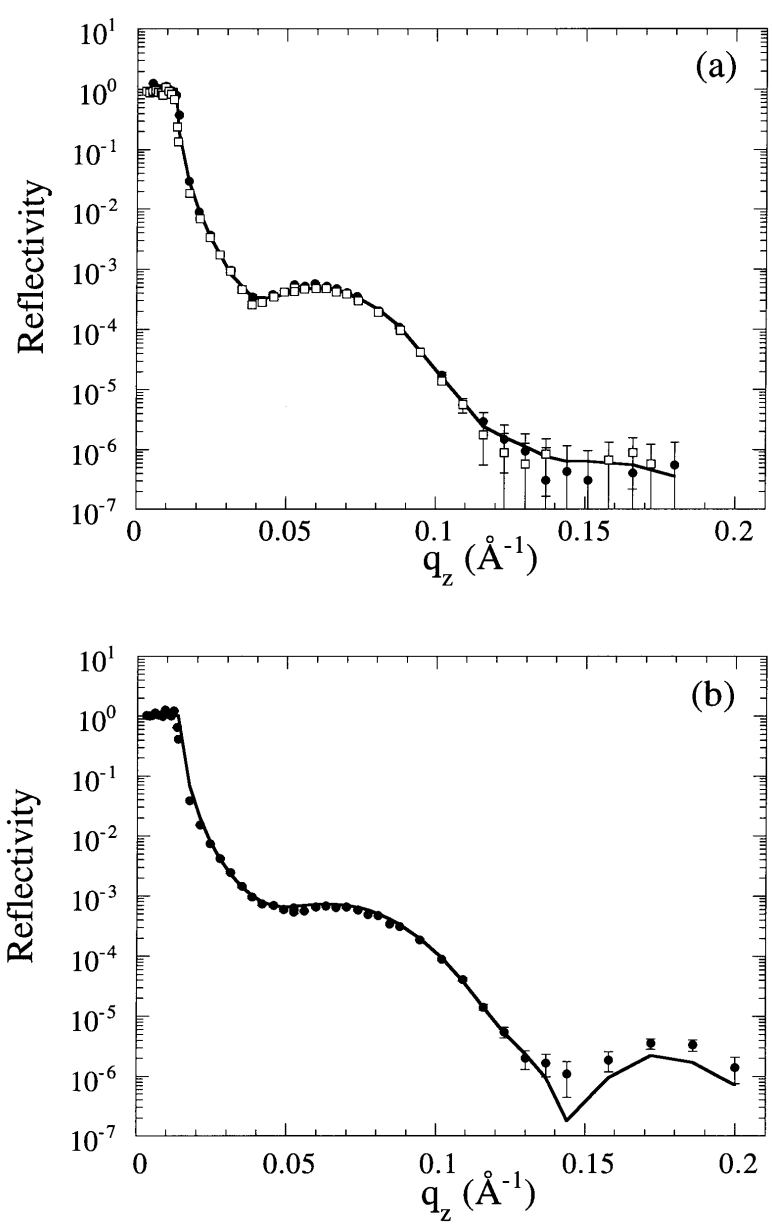

Fig. 7. Specular reflectivity profiles $R\left(q_{z}\right)$ of double-bilayers. (a) protonated DPPC in $87 \% \mathrm{D}_{2} \mathrm{O}:(\bullet)$ first measurement 24 hours after preparation, $(\square)$ second measurement of the same sample 48 hours after preparation; (b) protonated DSPC in $\mathrm{D}_{2} \mathrm{O}$. Solid lines are best fits by parameters listed in Table 3.

- This structure is stable: there was no observable difference between both DPPC samples.

- The second bilayer contained a proportion of water similar to the first, within an error bar of $3 \%$ : namely $18 \%$ versus $15 \%$ for DPPC, $17 \%$ versus $22 \%$ for DSPC. Since only one contrast was used, the amount of water and the roughness had similar effects on the fits and were hard to distinguish. In fact, it is a combination of both which is finely determined.

- The structure of the DSPC sample was the same as DPPC but for a $2 \AA$ increase in the thickness of the tails boxes.

- The water film between the substrate and the first bilayer was no longer observed.

- Both DPPC and DSPC bilayers were $2 \AA$ thicker than in the case of the single bilayer.

\section{Discussion}

\subsection{Structure of the layers}

The measured value of the DPPC bilayer thickness, $49 \pm$ $1 \AA$, agrees with literature $[11,24]$. The thickness of the tail box $(32 \pm 1 \AA)$ is slightly lower than twice the length of a stretched 16-carbons chains, namely $1.5+1.265 \times 16=$ $21.74 \AA[25]$. This suggests that chains are tilted with respect to the normal of the substrate. The tilt angle is ar$\operatorname{ccos}(32 / 43.5) \approx 40^{\circ}$, which is reasonable [24] for a bilayer deposited at $30 \mathrm{mN} / \mathrm{m}$. This value also agrees with neutron diffraction on DPPC multibilayers [26]. The thickness of DSPC tail region is compatible with the same molecular tilt. For both DPPC and DSPC double bilayers the tilt is slightly smaller, but not significantly; the higher surface pressure might have led to more compact, less tilted chains.

The water film between single bilayers and the substrate is observed by a non-perturbative in situ method. It is unambiguously distinguished from the thin, dry silica layer. Such distinction is impossible in lower resolution experiments [11] unless the film is thicker, for instance on a $17 \AA$ rough quartz substrate [24]. Here, on a smooth substrate, we find that the film is $7-8 \AA$ thick. Since the sum of all box thicknesses (silica+water film+bilayer) is derived directly from reflectivity data, independently of the model used in the fits, no other model could yield a thickness very different from this value. This low value, probably due to an optimal pulling speed of deposition, is close to the expected equilibrium value due to the hydration of lipid heads and substrate, both being highly 
hydrophilic [27]. Higher deposition speeds might result in an incomplete water drainage, the thickness of the film being determined by hydrodynamic dissipation [20].

For the double bilayers, there were more parameters in the fit but error bars on thicknesses were still between 0.5 and $1 \AA$. As already mentioned, the sum of all box thicknesses is model-independent. Thus no model can fit the reflectivity curves unless it includes a total thickness of water of order $20-30 \AA$. This seems to be the thickness of the second water film, namely the hydration film lying between both bilayers. The first water film, not visible, might well be much thinner than for single bilayers and certainly is not thicker; this discrepancy might be due to the rougher substrates and the higher deposition surface pressure.

The measured roughness and homogeneity of the silicon were conform to expectations and optical measurements. On the other hand, with the UV-ozone treatment the oxide layer was surprisingly good: low value of thickness and great homogeneity. We systematically looked for water contamination, which is usually found in oxides grown in aqueous solutions [10], but did not detect any. The same silicon blocks, without lipids, have been investigated in four water contrasts [13]: all results indicate the same roughness, thickness and homogeneity of oxide. The roughness measurement was validated on a block which had $50 \AA$ roughness and clearly visible Yoneda peaks.

\subsection{Coverage rates}

The controlled deposition of single PC bilayers at $30 \mathrm{mN} / \mathrm{m}$, or double PC bilayers, has been possible thanks to a combination of Langmuir-Blodgett and LangmuirSchaeffer techniques. Using either technique separately has always failed, to our knowledge $[18,22]$. LangmuirBlodgett deposition of more than three PC monolayers requires in the subphase ions such as uranyl salts [28] which are banned for biophysical experiments. The LangmuirSchaeffer method enables depositions far from equilibrium even in energetically not favourable configurations [3].

During the deposition of a single bilayer, the lipids of both monolayers are likely to mix to protect hydrophobic tails from water [4]. This quick rearrangement is different from the (slower) flip-flop within an already formed bilayer. The substrate surface is covered by zero or two layers, nowhere by one layer $[4,19]$. Thus, with global transfer rates of 0.97 and 0.9 , we can infer that a single bilayer covers $93 \%$ of the substrate. For double bilayers, we obtain $83 \%$ for the first bilayer and $93 \%$ for the second one; we do not know whether lipids of both bilayers mix. The instantaneous transfer rates, which are independent on assumptions on the geometrical corrections, yield estimations even closer to $100 \%$. On the other hand, measurements after deposition yield lower estimations. For single bilayers, neutron reflectivity, confirmed by atomic force microscopy, indicates that $80-85 \%$ of the substrate is covered by a smooth bilayer. This discrepancy might be interpreted as an in-plane reorganisation of molecules after deposition, for instance due to mechanical constraints.

\subsection{Neutron reflectivity resolution}

We hope to have demonstrated that specular neutron reflectivity is now fully adapted for a non-invasive investigation of the structure, hydration and fluctuations of soft and thin bilayers. Thicknesses as low as $4 \AA$ can be measured within $1 \AA$ precision and sensitivity. Measurements of head and tail sizes, normal to their plane, were compatible with, but more precise than values previously published [11,24]. In particular, our results confirm that, as Koening et al. demonstrated [11], the modelisation of bilayers by stacked boxes is valid.

In order to save beam time, neutron reflectivity is routinely performed at constant relative angular resolution $\Delta \theta / \theta$ : opening the slits at large $\theta_{\text {in }}$ increases the flux. However, this overestimates reflectivity at growing values of the momentum transfer $q_{z}$ : since resolution decreases, the off-specular reflection increasingly contributes to the apparently specular peak. Thus this method is valid only when the roughness, $\sigma$, is much smaller than $q_{z}^{-1}[7,29]$. We experimentally checked, for single bilayers deposited at surface tensions above $30 \mathrm{mN} / \mathrm{m}$, that $q_{z} \sigma \ll 1$ at least for $q_{z}<0.25 \AA^{-1}$. In the case of double bilayers, or of proteins inserted in the bilayers, this assumption reached its validity limit and we had to keep $\Delta \theta_{i n}$ constant and small.

Improvements concerned not only this question of angular divergence and other geometrical parameters of the set-up, but also: sample preparation; extraction of significant values of specular reflectivity $R\left(q_{z}\right)$ as low as a few $10^{-7}$, from a diffuse off-specular background at a signal/noise ratio below 0.1 ; simultaneous fitting of up to four $R\left(q_{z}\right)$ curves for different water contrasts. It had been suggested [11] that high quality neutron reflectivity data on deposited phospholipid bilayers can be obtained only if the water reservoir is much thinner than the penetration length of neutrons in water (about $40 \mu \mathrm{m}$ ), and this in order to reduce the background coming from hydrogen incoherent scattering. In fact, our results are independent on the depth of water subphase. A much larger water reservoir (300 $\mu \mathrm{m}$ or $1 \mathrm{~mm})$ gave the same quality data than measurements done in $5 \mu \mathrm{m}$ thick water (unpublished data), with a lower risk of damaging the bilayer during manipulations.

\section{Conclusions}

Deposition of lipid bilayers on solid substrates has been optimised for their use as artificial assays of membranesolid or membrane-protein interaction. Deposition of two monolayers ("single bilayer") has resulted in an adsorbed bilayer; deposition of two additional monolayers ("double bilayer") has resulted in a new type of free bilayer floating 20 to $30 \AA$ above the first one.

\subsection{Properties of adsorbed bilayers}

Thanks to the Langmuir-Schaeffer technique, out-ofequilibrium phosphocholine bilayers at the silicon-water 
interface could be reproducibly prepared at a deposition pressure much below the collapse, i.e. $30 \mathrm{mN} / \mathrm{m}$, with a reproducible coverage rate around $80 \%$. Bilayers were also deposited at surface pressure as low as $15 \mathrm{mN} / \mathrm{m}$, with the same structure but a coverage rate of $50 \%$ (unpublished data). Bilayers are stable over days and we have evidenced three marked advantages over layers deposited from vesicles:

(i) Bilayers have the expected structure: they have actually two and only two layers;

(ii) They are at a fixed distance from the substrate. A water film lies in-between: it is clearly resolved and its thickness seems to be at its equilibrium value;

(iii) They are flat and ordered.

\subsection{Properties of free bilayers}

The free bilayer at the solid-liquid interface is a new system with interesting advantages:

1. Over adsorbed bilayers at the solid-liquid interface [1]: it is more hydrated, since it is separated from the adsorbed bilayer by a $20-29 \AA$ thick film of water. It is thus more flexible, fluctuating, as evidenced in reflectivity by its measured roughness $(6-7 \AA$ more than the substrate) and the Yoneda peaks;

2. Over bilayers adsorbed from vesicles at the solid-liquid interface: it is more homogeneous and reproducible. It has the same ordered structure as a single bilayer deposited from a Langmuir monolayer; measured head and tails thicknesses are the same, but for a slight increase in tails thicknesses (decrease in tilt) at higher deposition pressure;

3. Over bilayers at the air-water interface [9]: it is robust. It is easy to create with a standard Langmuir-Blodgett trough. A clean environment is preferable, but no control of the atmosphere or temperature is strictly necessary. They resist manipulation, transport and storage for days as long as they are kept in hydrophilic media;

4. Over multilamellar systems: it has a well known position defined by the substrate. This makes possible its study with local investigation techniques.

5. Over bilayers suspended at the water-water interface [30]: it is homogeneous and can extend over square centimetres. Moreover it does not contain any spurious solvent or meniscus.

\subsection{Prospectives for soft matter physics}

In order to understand the stability and hydration of free bilayers, we are currently undertaking their complete characterisation, using off-specular reflectivity, different deuteration contrasts and even smoother substrates $\left(\leq 1.5 \AA \mathrm{rms}\right.$ over $\left.300 \times 300 \mu \mathrm{m}^{2}\right)$. Moreover, we will carry on the investigation of:

(i) the effective surface tension of single and double bilayers, in relation with the surface pressure of the monolayers before deposition; and (ii) the entropic effect of steric hindrance by the wall on the fluctuations of an individual free bilayer [31].

Free bilayers open interesting perspectives for bilayerbilayer interaction: a surface force apparatus could determine the force-distance relation between two identical free bilayers, each floating above a bilayer deposited on mica. Entropic polymer-bilayer interaction could be detected through the curvature induced by a polymer anchored in the bilayer [32]. What determines the thickness of the hydration film? The double bilayers have been deposited far from equilibrium, but their robustness suggests that they could be in a metastable state, resulting from the balance between energy minimisation and entropic repulsion. These speculations of course require further investigations. For instance, an increase in temperature could shift the balance towards a higher distance between bilayers, until they separate. We could envisage to associate one neutral and one charged bilayer; it is likely that two equally charged bilayers would repel each other. Other potentially interesting investigations concern asymmetric bilayers, where both monolayers have different chemical composition or surface tension, possibly yielding a spontaneous curvature, or an inhomogeneous charge repartition.

\subsection{Prospectives for biophysics}

Free and adsorbed bilayers are potentially interesting to investigate the interaction between soluble proteins and membrane lipids. Since more fluid bilayers are better models of cell membranes [33], it would be important to determine whether the fluidity in a free bilayer is higher or lower than in the monolayer before deposition. The effective surface tension of bilayers could be determined as mentioned in the preceding section; the lateral diffusion coefficients within their plane by fluorescence recovery after photobleaching.

Fluidity should be increased by using unsaturated lipids (such as DOPC) or mixed lipids rather than pure DPPC [3]. Neutron reflectivity has already been applied to detect the adsorption of a peptide into a bilayer of mixed phosphocholines and phosphatidyl-serines [13]. Increasing the temperature would increase the fluidity too but could result in the detachment of the free bilayer. Similarly, a lower surface pressure is supposed to increase the fluidity; for instance, at the air-water interface, the same peptide was shown to insert much better in a monolayer at 15 than at $30 \mathrm{mN} / \mathrm{m}$. However, after deposition at the solid-liquid interface, there is no indication of a similar effect, and the role of the deposition surface pressure is still unclear.

We would like to thank warmly those who helped us, especially A. Rennie, R. Ghosh and P. Thirtle for their coupled fit software; S. Wood and the ILL staff for neutrons and environment; E. Paris for atomic force microscopy, P.-F. Lenne for ellipsometry, G. Lerondel for profilometry; A. Carminati, J.-L. Cheval and C. Sibourg for the construction of the Langmuir trough, M. Betton and A. Delconte for its computer control; P. Dubos, R. Paddon, L. Périno-Gallice, A. Perrier, G. Virone, 
for their active participation in the experiments; J. Daillant for support and stimulating discussions; R. Cubitt for reading the manuscript. G.F. benefits from a TMR grant from the European Union.

\section{Appendix: Optimisation of neutron reflectivity}

\section{Experimental protocol}

A reasonable compromise between angular resolution and flux, and hence duration of the experiment, was achieved with $\Delta q_{z} / q_{z}<5 \%$; since $\Delta \lambda / \lambda=1 \%$ that is $\Delta \theta_{i n}=4 \%$ of $\theta_{i n} \max \sim 0.15$ degree. Two special slits were separated by 1 metre: $S_{m}$ just after the monochromator and $S_{s}$ just before the sample. Each slit consisted of two $4 \mathrm{~cm}$ high vertical cylinders of boron nitrite placed on a horizontal rotation stage [34]. We chose to under-illuminate the sample as much as possible [35]. The constant $\Delta \theta_{\text {in }}$ was $0.06^{\circ}$ for $q_{z}<0.05 \AA^{-1}\left(S_{m}=1 \mathrm{~mm}\right.$ and $\left.S_{s}=85 \mu \mathrm{m}\right)$ and was $0.1^{\circ}$ at $q_{z}>0.05 \AA^{-1}\left(S_{m}=1 \mathrm{~mm}\right.$ and $\left.S_{s}=700 \mu \mathrm{m}\right)$. At $q_{z}=0.05 \AA^{-1}$ the scaling factor was determined with $1 \%$ precision by repeating the measurement with both slit configurations and averaging over all samples.

For $\mathrm{D}_{2} \mathrm{O}$ and $4 \mathrm{MW}$, curves obtained in 6-8 hours spanned 2 decades in $q_{z}$ and 7 in reflectivity. Reflected peaks were separated from the direct beam below their critical angles $\left(14 \times 10^{-3}\right.$ and $\left.8 \times 10^{-3} \AA^{-1}\right)$, down to $3.710^{-3} \mathrm{~A}^{-1}$ [35]. Due to the restriction of wavelength and of constant $\Delta \theta_{i n}$, which decreases the neutron counting rate like $q_{z}^{-4}$, each high- $q_{z}$ point had to be counted 40 minutes; the reflected peak could be extracted from noise up to $0.25 \AA^{-1}$.

For SMW and $\mathrm{H}_{2} \mathrm{O}$ contrasts, curves obtained in 9-10 hours spanned a factor 30 in $q_{z}$ and 6 decades in reflectivity. The direct and reflected beams were separated for $\theta_{i n}>10^{-2}$ degree. Due to an increased background, the signal-to-noise ratio at high- $q_{z}$ was correct up to $\theta_{\text {in }}<3$ degrees.

\section{Data reduction}

The background was due to the incoherent scattering, mainly of the hydrogen atoms of the liquid medium, which is constant over the angular range; to the instrument noise, very weak in this case, of the order of 3-5 neutrons/sec over the whole detector; and to the diffuse scattering caused by the roughness of the sample and assumed to be small and proportional to $q_{z}^{-2}[7,29]$ (it is constant for $q_{z} \sim \sin \theta_{\text {in }}+\sin \theta_{\text {out }}=$ constant, but not for $\theta_{\text {in }}=$ constant [36]). The overall background was fitted by a second order polynom in $\theta_{\text {in }}$ over twenty points around the peak (except near the direct beam). As expected, the three fitting parameters correlate to the size of the beam footprint and to the substrate roughness, but not to the deposited lipid layers.

The variations in $\theta_{\text {in }}$ were chosen to correspond to an integer number of wires. The reflected peak, with its maximum intensity falling on one wire and symmetric intensities on the next wire on both sides, was best fitted [36] by a Gaussian. Its position was checked to coincide with $\theta_{i n}$. Its width, determined by the experimental geometry, was constant within the experimental error and therefore was fixed to its mean value HWHM $=8 \times 10^{-2}$ degrees. The intensity of the Gaussian was thus the only free parameter; its error accounts for the statistical error of each point, the data discretisation (less than a few percents), and the sensitivity of the fit. The specular intensity $\sim q_{z}^{-4}$ decreases more quickly than the background $\sim q_{z}^{-2}$, nevertheless it could be extracted at a signal to noise ratio below 0.1 .

\section{Fitting the reflectivity curves}

The values of all parameters were initially set according to our knowledge of the sample and published data $[11,33]$. Each parameter could be either fixed, or varied (freely or linked to another parameter) thanks to the programs WETDOC [37] and AFIT [38] which use the Abeles method [39], a modified optical matrix method. The sum of the unweighted square deviation between the calculated and the experimental (logarithm of) values was calculated not only for each single curve, but cumulated on all curves of the different deuteration contrasts which were modelled simultaneously. This probed the local and global shape of the minimisation landscape: presence and depth of local minima, importance of each parameter, influence of their coupled variations. In addition, manual fitting assayed by eye proved more sensitive to qualitative features of curves such as: inflexion points, systematic deviations, curvature, experimental error bars, balance between the fit at small and large wavevectors.

The number of boxes was itself the first parameter. For single bilayers, systematic trials and extensive tests a posteriori with different number of boxes showed that four boxes or less could not result in any correct fit, while six boxes or more resulted in unconstrained fits, i.e. a fitting landscape with no pronounced minimum; with five boxes, there was systematically a rapid and robust convergence of the parameters towards one and only one optimum fit. For double bilayers, we never detected the water layer between the substrate and the first bilayer, and the existence and unicity of the fit was observed for 8 and only 8 boxes.

Four parameters were associated with each box: the average thickness; the neutron scattering length density of the box without solvent; the root mean square of the interface roughness; and the amount (percentage vol:vol) of solvent in the box, a contribution to the refraction index which is linearly correlated with the water contrast. In practice, for a single bilayer, our fits evidenced the really independent parameters: thickness of the water film; scattering length and thickness of heads and tails; amount of water in the bilayer. The water, head, tail and head boxes had roughnesses, if not exactly equal, at least nearly in growing order. Double bilayers were fitted using 10 independent parameters: the thickness of silica, heads, tails, water (4 parameters); the amount of water in each bilayer (2 parameters); the roughness (4 parameters in growing 
order, thus not strictly independent). All assumptions derived from successive refinements of the fits were checked a posteriori by comparing fits with different seeds, different update sequences, several samples with the same lipids, similar samples with different lipid deuterations.

\section{References}

1. E. Sackmann, Science 271, 43 (1996).

2. G. Roberts, Langmuir Blodgett Films (Plenum Press, New York 1990).

3. L.K. Tamm, H.M. McConnell, Biophys. J. 47, 105 (1985).

4. S. Alexandre, N. Dubreuil, C. Fiol, J.-J. Malandain, F. Sommer, J.-M. Valleton, Microsc. Microanal. Microstruct. 5, 359 (1994).

5. J.R. Lu, E.M. Lee, R.K. Thomas, Acta Cryst A 52, 11 (1996).

6. J. Penfold et al., J. Chem. Soc. Far. Trans. 93, 3899 (1997).

7. J. Daillant, O. Belorgey, J. Chem. Phys, 97, 5824 (1992).

8. J. Als-Nielsen, in Neutron and synchrotron radiations for condensed matter studies, Hercules Course, edited by J. Baruchel, J.L. Hodeau, M.S. Lehmann, J.R. Regnard, C. Schlenker (Les Éditions de Physique-Springer, 1993) Vol. 1, p. 3.

9. N.L. Gershfeld, K. Tajima, Nature 279, 708 (1979); K. Fukada, N.L. Gershfeld, J. Phys. Chem. B 101, 8225 (1997).

10. G. Fragneto, J.R. Lu, D.C. McDermott, R.K. Thomas, Langmuir 12, 477 (1996).

11. B.W. Koening, S. Krueger, W.J. Orts, C.F. Majkrzak, N.F. Berk, J.V. Silverton, K. Gawrisch, Langmuir 12, 1343 (1996).

12. J. Penfold, R.K. Thomas, J. Phys.-Cond. Matter 2, 1369 (1990).

13. G. Fragneto, F. Graner, T. Charitat, P. Dubos, E. Bellet-Amalric, to appear in Biophys. J. (1999).

14. D.S. Silvia, W.A. Hamilton, G.S. Smith, Physica B 173, 121 (1991).

15. N.F. Berk, C.F. Majkrzak, Phys. Rev. B 51, 11296 (1991).

16. J.R. Williams, A.M. Goodman, Appl. Phys. Lett. 24, 531 (1974).

17. J.R. Vig, Vac. Sci. Technol. A 3, 1027 (1984).

18. T.D. Osbom, P. Yager, Biophys. J. 68, 1364 (1995).

19. P. Bassereau, F. Pincet, Langmuir 13, 7003 (1997).
20. C. Mingotaud, P. Bassereau, F. Pincet, private communication.

21. A. Lambacher, P. Fromherz, Appl. Phys. A 63, 207 (1996).

22. H. Hasmonay, M. Caillaud, M. Dupeyrat, Biochem. Biophys. Res. Commun. 89, 338 (1979).

23. Y.Yoneda, Phys. Rev. 131, 2010 (1963).

24. S.J. Johnson, T.M. Bayerl, D.C. McDermott, G.W. Adam, A.R. Rennie, R.K. Thomas, E. Sackmann, Biophys. J. 59, 289 (1991).

25. C. Tanford, J. Phys. Chem. 76, 3020 (1972).

26. G. Büldt, H. Gally, A. Seelig, J. Seelig, G. Zaccai, Nature 271, 182 (1978).

27. J. Israelachvili, Intermolecular and surface forces (Academic Press, London 1985).

28. J. Peng, M. Prakash, R. Macdonald, P. Dutta, J. Ketterson, Langmuir 3, 1096 (1987).

29. J.P. Rieu, Thesis, Grenoble University, unpublished, 1996.

30. M. Montal, P. Mueller, Proc. Natl. Acad. Sci. USA 69, 3561 (1972).

31. W. Helfrich, Z. Naturforsch. 30c, 841 (1975).

32. R. Lipowsky, H.-G. Döbereiner, C. Hiergeist, V. Indrani, Physica A 249, 536 (1998).

33. A. Tardieu, V. Luzzati, F. Reman, J . Mol. Biol. 75, 711 (1973).

34. T. Charitat, Thesis, Grenoble University, unpublished, 1997.

35. The correction to over-illumination (when the beam footprint is larger than the sample projection) would have required a detailed knowledge of the beam parameters, and thus a fully dedicated reflectometer (C. Fermon, private communication). Here over-illumination is restricted to $\theta_{\text {in }}<1.5 \times 10^{-2}$ degrees. By averaging on all the curves from $\mathrm{D} 2 \mathrm{O}$ contrast we obtain a mean geometric linear correction in this $\theta_{i n}$ range. This normalisation yields the good critical angle for $\mathrm{D}_{2} \mathrm{O}$ and $4 \mathrm{MW}$ contrasts so we assumed it valid for the other contrasts.

36. The reflectivity $R\left(\theta_{\text {in }}, \theta_{\text {out }}\right)$ was fitted along a curve $\theta_{\text {in }}=$ constant, and not along $q_{z}=$ constant as it should be. Since $\Delta \theta_{i n}$ was kept constant and small, so was the resulting error.

37. Wetdoc 1.0: coupled fit programm specially written by A. Rennie (Cambridge University) as a modification of Drydoc 3.2 (A. Rennie) which uses the Fitfun library (R. Ghosh, ILL), see http://www.ill.fr/Computing.

38. Afit: coupled simulation programm written by Thirtle (Oxford University), see http://www.ill.fr/Computing.

39. J. Penfold, report 88-088, Rutherford Appleton Laboratory, Chilton, Didcot, Oxon OX11 0QX, UK (1988). 